\title{
Neutralização da acidez em substrato de casca de pinus com diferentes granulometrias
}

Fernanda Ludwig', Dirceu Maximino Fernandes², Amaralina Celoto Guerrero 3 , Guilherme Amaral Ferreira ${ }^{4}$, Valeria Pohlmann ${ }^{5}$

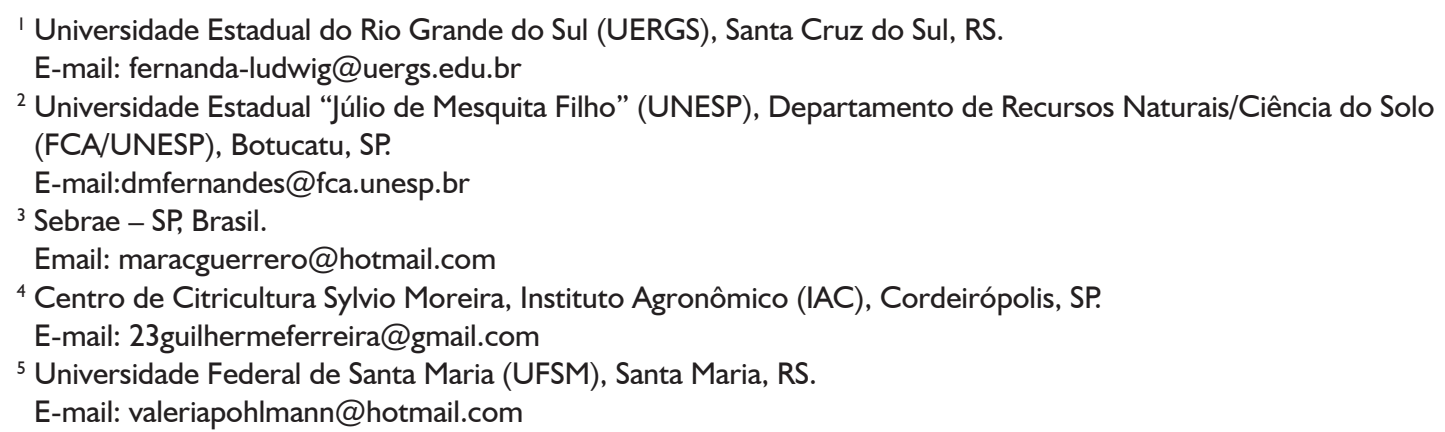

\section{Resumo}

Os substratos são utilizados na maioria dos cultivos em plantas ornamentais. Entre suas características essenciais, destaca-se o valor do potencial hidrogeniônico $(\mathrm{pH})$, que pode influenciar a disponibilidade de nutrientes às plantas, podendo favorecer sua absorção, dificultar e ainda causar fitotoxidez. Apesar da sua importância, as informações que auxiliem na sua manutenção para adequar às necessidades das plantas são escassas. Desse modo, o presente trabalho foi conduzido com o objetivo de avaliar a neutralização da acidez em substrato de casca de pinus com diferentes granulometrias. $O$ experimento foi conduzido em casa de vegetação, em delineamento experimental de blocos ao acaso com 5 doses de calcário dolomítico (5, 10, 15, 20 e $\left.25 \mathrm{~g} \mathrm{~L}^{-1}\right), 5$ granulometrias de casca de pinus (<4mm; 4-2mm; <2mm; 2-I mm; $<$ Imm), 4 tempos de incubação ( $10,20,30$ e 40 dias) e 4 repetições. Os valores de $\mathrm{pH}$ da solução do substrato foram determinados a cada 10 dias, utilizando o método de diluição do $1: 5$. Os valores de $\mathrm{pH}$ do substrato diferiram entre as diferentes granulometrias, doses de calcário e dias de incubação. As menores granulometrias, de $<\mathrm{Imm}$ e de I-2mm apresentaram maiores valores de $\mathrm{pH}$. $\mathrm{O}$ aumento das doses de calcário resultou em aumento do $\mathrm{pH}$, enquanto o aumento do tempo de incubação, resultou em decréscimo do $\mathrm{pH}$. Os substratos com diferentes granulometrias de casca de pinus devem receber quantidades de calcário distintas para adequar os valores de $\mathrm{pH}$. Não se recomenda utilizar o substrato na primeira semana após a aplicação do calcário devido à instabilidade do $\mathrm{pH}$, independente da granulometria.

Palavras-chave: Cultivo sem solo. Floricultura. Incubação do calcário. Nutrição.

\section{Abstract}

\section{Acid neutralization in pine bark substrate with different grain size}

The substrates are used in most crops in ornamental plants. Among its essential characteristics, it is worth highlighting the value of the hydrogen-ionic potential $(\mathrm{pH})$, which can influence the availability of nutrients to the plants, which can favor its absorption, hinder and still cause phytotoxicity. Despite its importance, the information that helps to maintain it to suit the needs of plants is scarce. Thus, the present work was conducted with the objective of evaluating the neutralization of the acidity in substrate of pinus bark with different granulometry. The experiment was conducted in a greenhouse in a randomized block design with 5 doses of dolomitic limestone (5, 10, 15, 20 and $\left.25 \mathrm{~g} \mathrm{~L}^{-1}\right), 5$ pinus bark granulometry (<4 mm, 4-2 mm, 2-I mm, <I mm), 4 incubation times (10, 20, 30 and 40 days) and 4 replicates. The $\mathrm{pH}$ values of the substrate solution were determined every 10 days using the $1: 5$ dilution method. The $\mathrm{pH}$ values of the 
substrate differed between the different particle sizes, limestone doses and incubation days. The smaller particle sizes of $<\mathrm{Imm}$ and $\mathrm{I}-2 \mathrm{~mm}$ presented higher $\mathrm{pH}$ values. Increasing the doses resulted in increased $\mathrm{pH}$, while increasing the incubation time, resulted in a decrease in $\mathrm{pH}$. Substrates with different pine bark granulometry should receive different amounts of limestone to adjust the $\mathrm{pH}$ values. It is not recommended to use the substrate in the first week after application of limestone due to $\mathrm{pH}$ instability.

Keywords: Cultivation without soil. Floriculture. Incubation of limestone. Nutrition.

\section{Introdução}

O sistema de produção em substratos vem sendo empregado na maioria dos cultivos comerciais de plantas ornamentais (LUDWIG et al., 20I4). A escolha de um substrato adequado ao cultivo de plantas em vaso requer o prévio conhecimento das suas características químicas e físicas para melhor formulação de misturas, adubação e irrigação (LUDWIG et al., 20l0). As diferenças em suas características ocorrem em função da origem, método de produção ou obtenção e proporções de seus componentes (KRATZ et al., 20I3). Apesar da criação da legislação brasileira regulamentar a comercialização de substratos (BRASIL, 2007), este assunto ainda é considerado novo no Brasil e requer mais estudos.

A demanda por substratos tem aumentado, em diversos setores do agronegócio. A utilização de alguns resíduos poderia ser a solução para essa cadeia produtiva, minimizando assim o impacto ambiental que possam gerar. A casca de pinus é um exemplo de resíduo que é amplamente utilizado como substrato tanto na forma pura, como na variação da sua granulometria ou ainda na formulação de substrato juntamente com outros materiais (LONE et al., 2008; SAMPAIO et al., 2008; KRATZ; WENDLING, 20I3; KRATZ et al., 20I3).

A disponibilidade de nutrientes é um dos principais fatores que influenciam a adequação de substratos orgânicos para o crescimento das plantas, o que depende não somente da presença dos nutrientes, mas também da capacidade de adsorção, do potencial hidrogeniônico $(\mathrm{pH})$, da estabilidade biológica e da presença de compostos orgânicos dissolvidos (CABALLERO et al., 2007).

Para a maioria das culturas ornamentais o $\mathrm{pH}$ ótimo do substrato situa-se entre 5,4 e 6,3 (BAILEY; BILDERBACK, 1997; HANDRECK; BLACK, 2002). Segundo Cavins et al. (2000), o pH é mais importante que a própria nutrição do substrato, pois afeta principalmente a absorção dos micronutrientes, tais como ferro $(\mathrm{Fe})$ e manganês $(\mathrm{Mn})$, que em valores de $\mathrm{pH}$ abaixo de 5,8 tem sua disponibilidade aumentada, podendo levar a uma toxidade para muitas espécies sensíveis ao baixo $\mathrm{pH}$.

De acordo com Handreck e Blanck (2002), o Mn é tóxico principalmente porque desintegra as células da raiz e folha, pelo aumento da atividade da peroxidade, resultando em pequenas pontuações escuras e necróticas, com tamanho e distribuição irregular pelas folhas.

O manganês é requerido para atividade da peroxidase (TAIZ; ZEIGER, 2009) e a toxidez tem sido associada ao aumento da atividade desta enzima, resultando no escurecimento necrótico em folhas (HORIGUCHI; FUKUMOTO, 1987, apud EL-JAOUAL; COX, 1998). Pequenas pontuações escuras e necróticas, com tamanho e distribuição irregular pelas folhas tem sido relatadas como sintomas de toxidez de $\mathrm{Mn}$ em plantas de gérbera (LUDWIG; GUERRERO; FERNANDES, 20I4; MERCURIO, 2002).

Em valores de $\mathrm{pH}$ elevados, alguns nutrientes essenciais para o crescimento de plantas tornam-se indisponíveis e, portanto, os sintomas de deficiência começam a surgir, sendo a deficiência de Fe a mais comum. A clorose ocasionada pela deficiência de Fe é o maior problema nas plantas cultivadas em substratos com pH alto, caracterizado pelo amarelecimento das folhas novas, em contraste com o verde frequentemente encontrado nas folhas maduras (CABALLERO et al., 2007), característica essa observada em plantas de gérbera conduzidas com substrato com pH superior a 7,0 (LUDWIG et al., 20I4).

A importância do conhecimento dos valores de $\mathrm{pH}$ está relacionada com sua influência na disponibilidade de nutrientes bem como no efeito sobre processos fisiológicos da planta (KÄMPF, 2000). No momento da formulação dos substratos, o $\mathrm{pH}$ pode ser ajustado pela aplicação de soluções ácidas ou pela mistura de componentes de $\mathrm{pH}$ mais baixo (KRATZ et al., 20I3). Apesar da sua importância, as informações que auxiliem na manutenção do $\mathrm{pH}$ para adequar às necessidades das plantas são escassas (BOARO et al., 20l4).

Entre as práticas para a correção da acidez do solo, o uso de calcário é a mais utilizada, pois além de regular o $\mathrm{pH}$, fornece nutrientes como cálcio $(\mathrm{Ca})$ e magnésio $(\mathrm{Mg})$, aumenta a disponibilidade de fósforo (P) e reduz a toxidez por alumínio (Al) no solo (CAIRES et al., 2008; CHAVES; FARIAS, 2008; ARAÚJO; 
DEMATTÊ; GARBUIO, 2009; POLETTO et al., 20II), sendo que as mesmas também podem ser utilizadas para o ajuste de $\mathrm{pH}$ no substrato. Problemas decorrentes dos desbalanços nos valores de $\mathrm{pH}$ são evitados com a determinação inicial dessa propriedade nos substratos e correção antes do início do cultivo, pois os erros iniciais da calagem podem não ser revertidos a tempo, devido ao maior poder tampão dos substratos orgânicos, dificultando a correção no $\mathrm{pH}$.

Os componentes do substrato, sua granulometria e características físicas e químicas relacionadas, afetam inicialmente a disponibilidade de nutrientes e a salinidade do meio de cultivo e a escolha deverá considerar a necessidade inicial da cultura, bem como sua tolerância a salinidade (LUDWIG; GUERRERO; FERNANDES, 2014). A caracterização física e química dos substratos é necessária para a sua correta formulação e, também, para a recomendação e monitoramento das adubações nos sistemas de cultivo protegido (ABREU et al., 2007), determinando a melhor qualidade do produto final (FERMINO, 2003). Desse modo, o presente trabalho foi conduzido com o objetivo de avaliar doses de calcário na neutralização da acidez do substrato a base de casca de pinus separado em diferentes granulometrias.

\section{Material e Métodos}

O experimento foi desenvolvido em condições de casa de vegetação, no Departamento de Recursos Naturais/Ciência do Solo da FCA/UNESP, no município de Botucatu (22 $50^{\prime}$ 'S e $48^{\circ} 26^{\prime} \mathrm{W}$ ), estado de São Paulo, no período de maio a junho de 2009. O delineamento experimental utilizado foi o de blocos ao acaso em esquema fatorial $5 \times 4$ (doses de calcário e tempo de incubação) e 4 repetições, em substrato de casca de pinus, separada nas granulometrias de $<4 \mathrm{~mm}, 4-2 \mathrm{~mm},<2 \mathrm{~mm}, 2-\mathrm{Imm},<\mathrm{Imm}$.

Os substratos foram caracterizados fisicamente quanto à densidade úmida e seca (BRASIL, 2007) e curva de retenção de água (BOODT; VERDONCK, 1972) e quimicamente quanto ao $\mathrm{pH}$, condutividade elétrica pelo método da diluição do I:5 (BRASIL, 2007). Os resultados das análises para os substratos são apresentados na Tabela I.

Tabela I - Características físicas e químicas dos substratos com diferentes granulometrias utilizados no experimento.

\begin{tabular}{cccccccccc}
\hline \multirow{2}{*}{ Substratos } & DU & DS & PT & S & EA & AD & AR & CE & \multirow{2}{*}{ PH } \\
\cline { 2 - 8 } & \multicolumn{2}{c}{$\mathrm{kg} \mathrm{m}^{-3}$} & & & $\%$ & & & \multicolumn{1}{c}{$\mathrm{dS} \mathrm{m}^{-1}$} & \\
\hline$<4 \mathrm{~mm}$ & 450 & 350 & 83,1 & 16,9 & 25,7 & 31,4 & 26,0 & 0,69 & 4,5 \\
$4-2 \mathrm{~mm}$ & 340 & 250 & 82,4 & 17,6 & 41,6 & 16,3 & 24,5 & 0,47 & 4,5 \\
$<2 \mathrm{~mm}$ & 420 & 340 & 80,6 & 19,4 & 16,8 & 39,7 & 24,1 & 0,67 & 4,6 \\
$2-1 \mathrm{~mm}$ & 360 & 300 & 80,9 & 19,1 & 35,0 & 21,2 & 24,7 & 0,52 & 4,6 \\
$<1 \mathrm{~mm}$ & 440 & 400 & 79,8 & 20,2 & 7,9 & 49,6 & 22,3 & 0,65 & 4,6 \\
\hline
\end{tabular}

DU: densidade úmida; DS: densidade seca; PT: porosidade total; S: sólidos; EA: espaço de aeração; AD: água disponível; AR: água remanescente; CE: condutividade elétrica; pH: potencial hidrogeniônico. Fonte: Botucatu, SP. 2009.

As doses de calcário dolomítico, com PRNT de 92\% foram de 5, 10, 15, 20 e $25 \mathrm{~g} \mathrm{~L}^{-1}$. Utilizou-se o vaso com volume de I L (II,5 cm de altura, $13 \mathrm{~cm}$ de base superior e $9 \mathrm{~cm}$ de base inferior), preenchido com base na densidade úmida dos substratos. A quantidade de calcário referente aos tratamentos foi adicionada à massa de substrato de cada vaso de forma individualizada, a fim de garantir uma melhor padronização e homogeneização na mistura. Após a aplicação do calcário, adicionou-se água ao substrato de modo a mantê-lo com $50 \%$ da água disponível, sendo então fechados com uso de saco plástico.

Após 10 dias de incubação do calcário (DAl) iniciou-se a análise de pH dos substratos, a qual foi repetida com intervalo de 10 dias, até os 40 DAl. Uma porção do substrato, de aproximadamente $100 \mathrm{~mL}$, foi retirada do vaso e deixada secar ao ar durante 24 horas a fim de padronizar a umidade das amostras, para posterior análise. A metodologia utilizada foi a diluição do I:5 (BRASIL, 2007), na qual tomou-se uma massa de amostra correspondente ao volume de $30 \mathrm{~mL}$, de acordo com a densidade, e transferiu-se para um frasco com tampa, adicionando 5 partes de água ( $150 \mathrm{~mL}$ ). A mistura foi agitada em agitador tipo 'Wagner' durante o período de uma hora, procedendo-se em seguida, a leitura do $\mathrm{pH}$.

Os resultados obtidos foram submetidos à análise de variância pelo teste $\mathrm{F}$. As médias dos substratos 
foram comparadas pelo teste Tukey a $5 \%$ de probabilidade. Os efeitos dos dias de incubação e das doses do calcário foram submetidos à análise de regressão, testando os modelos linear e quadrático, escolhidos com base na significância dos coeficientes de regressão a $1 \%(* *)$ e $5 \%\left({ }^{*}\right)$ de probabilidade pelo teste $\mathrm{F}$ e no maior valor do coeficiente de determinação $\left(R^{2}\right)$ e procedeu-se o desdobramento da interação entre as doses e os dias de incubação quando significativa. O programa estatístico utilizado foi o Sisvar (FERREIRA, 20I I).

\section{Resultados e Discussões}

Os valores iniciais de pH do substrato de casca de pinus foram de 4,5 e 4,6 (Tabela I), e a aplicação de $\mathrm{CaCO}_{3}$ foi efetiva para elevar os níveis à faixas adequadas ao desenvolvimento da maioria das culturas (Tabela 2). A disponibilidade de nutrientes é um dos principais fatores que influenciam a adequação de substratos orgânicos para o crescimento das plantas, o que está relacionado, sobretudo com o valor de pH da solução do meio (CABALLERO et al., 2007). Segundo Cavins et al. (2000), o pH é mais importante que a própria nutrição do substrato, pois afeta principalmente a absorção dos micronutrientes.

Tabela 2 - Valores médios de pH nos diferentes substratos e doses de calcário.

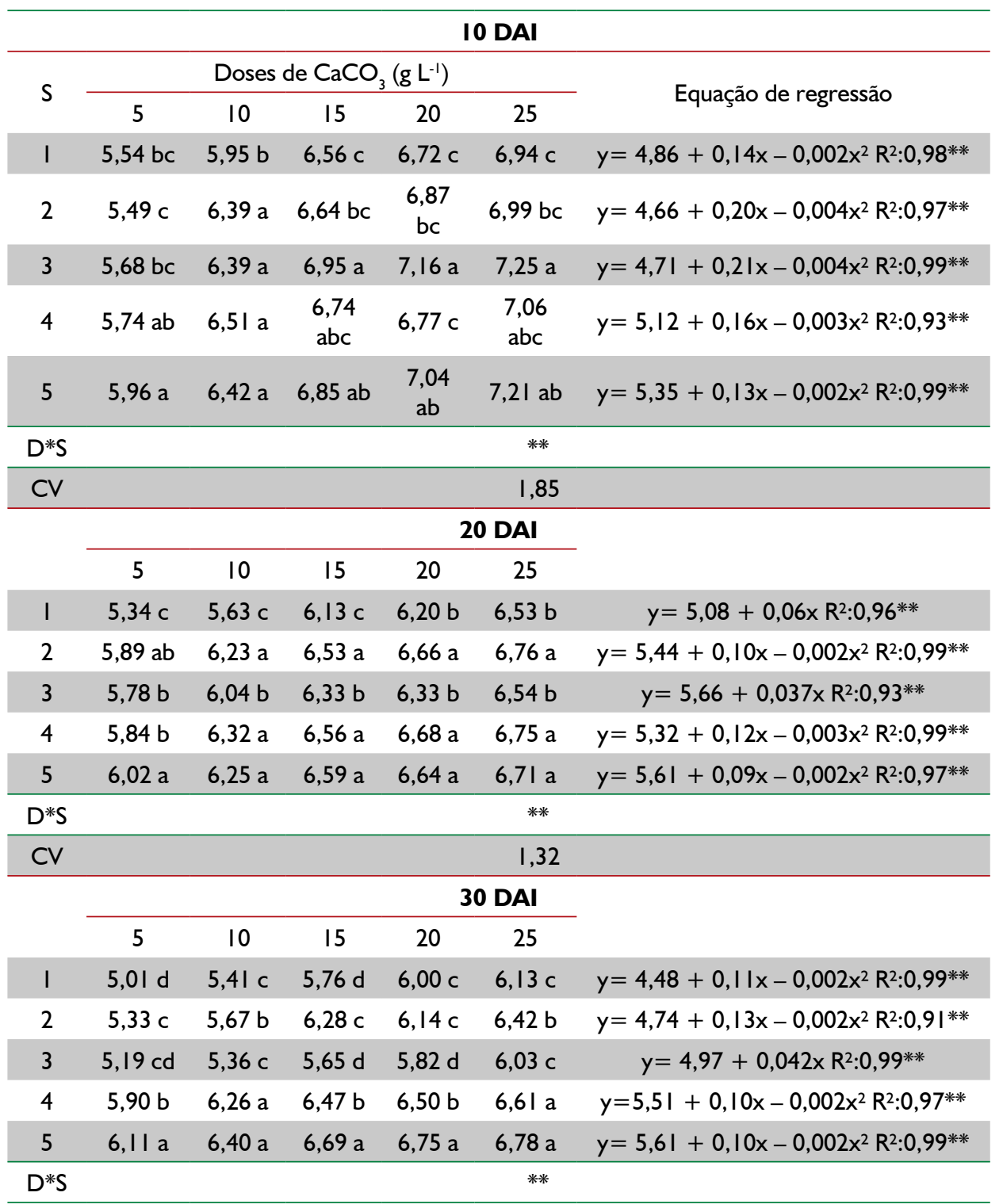




\begin{tabular}{|c|c|c|c|c|c|c|}
\hline \multirow[t]{3}{*}{$\mathrm{CV}$} & \multicolumn{6}{|c|}{$\mathrm{I}, 5 \mathrm{I}$} \\
\hline & \multicolumn{6}{|c|}{40 DAl } \\
\hline & 5 & 10 & 15 & 20 & 25 & \\
\hline 1 & $4,99 \mathrm{~b}$ & $5,65 b$ & $5,92 \mathrm{~b}$ & $6,19 a$ & $6,27 \mathrm{a}$ & $y=4,30+0,16 x-0,003 x^{2} R^{2}: 0,99 * *$ \\
\hline 2 & $5,20 \mathrm{~b}$ & $5,6 \mathrm{lb}$ & $6,02 \mathrm{ab}$ & $6,26 \mathrm{a}$ & $6,38 \mathrm{a}$ & $y=4,99+0,06 \times R^{2}: 0,95^{*} *$ \\
\hline 3 & $4,97 b$ & $5,26 c$ & $5,57 c$ & $5,86 b$ & $6,01 \mathrm{~b}$ & $y=4,72+0,054 x R^{2}: 0,98 * *$ \\
\hline 4 & $5,95 \mathrm{a}$ & $5,95 \mathrm{a}$ & $6,24 \mathrm{a}$ & $6,23 \mathrm{a}$ & $6,42 \mathrm{a}$ & $y=5,79+0,02 \times R^{2}: 0,89 * *$ \\
\hline 5 & $5,94 \mathrm{a}$ & $6,03 \mathrm{a}$ & $6,17 \mathrm{a}$ & $6,16 \mathrm{a}$ & $6,31 \mathrm{a}$ & $y=5,86+0,17 \times R^{2}: 0,99 * *$ \\
\hline D*S & & & & & **⿻丷木 & \\
\hline $\mathrm{CV}$ & & & & & 2,06 & \\
\hline
\end{tabular}

Médias seguidas da mesma letra na coluna não diferem entre si pelo teste de Tukey. NS: não significativo ao nível de $5 \%$ de probabilidade. ***** significativo a $5 \%$ e $1 \%$ de probabilidade. S: substratos. D: doses de $\mathrm{CaCO}_{3}$; S: substrato. I: $>4 \mathrm{~mm}, 2: 4-2 \mathrm{~mm}, 3:<2$ mm, 4: 2-I mm, 5: <I mm. Fonte: Botucatu, SP. 2009.

Os valores ótimos podem variar em função das espécies e cultivares, sendo necessário avaliar individualmente cada caso (HANDRECK; BLACK, 1999). Valores de $\mathrm{pH}$ inferiores a 5,0 podem promover deficiência de nutrientes como nitrogênio $(\mathrm{N})$, potássio $(\mathrm{K}), \mathrm{Ca}, \mathrm{Mg}$ e boro $(\mathrm{B})$ e valores de $\mathrm{pH}$ superiores a 6,5 podem resultar em problemas com a disponibilidade de P, Fe, Mn, zinco ( $\mathrm{Zn}$ ) e cobre (Cu) (KÄMPF, 2005). Substratos com pH entre 5,5 e 6,8 apresentaram adequada disponibilidade de nutrientes e forneceram condições satisfatórias para a produção de gérbera envasada, em trabalho desenvolvido por Ludwig et al. (2014). Também, Rogers e Tjia (1990) sugerem que o pH ideal para gérbera se situa entre 5,5 e 6,5.

Valores inadequados de $\mathrm{pH}$ podem afetar a disponibilidade de nutrientes, causando desequilíbrio fisiológico e afetando o desenvolvimento das plantas (ASSIS et al., 2005). Valores de $\mathrm{pH}$ entre 3,0 e 5,5 aumentam a disponibilidade de Mn, podendo levar a planta a toxicidade (MERCURIO, 2002). Ludwig et al. (20I4) verificaram sintomas de pequenas pontuações escuras e necróticas, com tamanho e distribuição irregular pelas folhas de gérbera em plantas conduzidas em substratos com valores de $\mathrm{pH}$ de 4,8. Por outro lado, substratos com valores de $\mathrm{pH}$ superiores a 7,0 tornam micronutrientes como $\mathrm{Fe}, \mathrm{Mn}$ e $\mathrm{Zn}$ menos disponíveis (LUDWIG et al., 20I4) e as plantas apresentaram sintomas de clorose (MERCURIO, 2002), devido à insuficiente absorção de Mn ou Fe (SONNEVELD; VOOGT, 1997).

Os valores de $\mathrm{pH}$ da solução do substrato elevaram-se em função do aumento da dose do $\mathrm{CaCO}_{3}$ aplicado, com respostas quadráticas e lineares, variando de 5,59 na dose de $5 \mathrm{~g} \mathrm{~L}^{-1}$ até 6,61 na dose de $25 \mathrm{~g} \mathrm{~L}^{-1}$ (Tabela 2). $\mathrm{O}$ aumento do $\mathrm{pH}$ com o aumento da quantidade de $\mathrm{CaCO}_{3}$ adicionada, se deve a uma maior quantidade de hidroxilas produzidas pelo ânion carbonato presente. Em estudo, Sousa et al. (20I3) também observaram o aumento do $\mathrm{pH}$ com o aumento das doses de $\mathrm{CaCO}_{3}$. Em aplicação de calcário no sulco da semeadura de feijão comum, Nascente e Cobucci (2015) também observaram aumento do pH do solo com o aumento das doses de $\mathrm{CaCO}_{3}$. O mesmo resultado também foi encontrado em Argissolo Vermelho alítico, em Latossolo Amarelo distrófico e em Espodossolo Humilúvico no estado do Acre (FRADE JUNIOR et al., 20 I3).

Houve redução nos valores de $\mathrm{pH}$ ao longo do tempo de incubação, com respostas quadráticas negativas (Tabela 3). $\mathrm{O}$ decréscimo do $\mathrm{pH}$ com o aumento no período de incubação demonstra o poder tampão do substrato, onde os íons $\mathrm{H}^{+}$são deslocados do complexo de troca pelo $\mathrm{Ca}^{2+}$ e, na solução do solo reagem com $\circ \mathrm{OH}$. Como a quantidade de hidroxilas adicionadas pelo calcário é limitada, ao serem utilizadas na neutralização da solução, com o alto poder tampão do substrato, a tendência durante o tempo, é a diminuição do $\mathrm{pH}$ do meio. Em estudo, Veloso et al. (1992) também verificaram a diminuição do pH com o período de incubação de calcário. 


\begin{tabular}{|c|c|c|c|c|c|c|c|}
\hline \multirow{2}{*}{ Substrato } & \multicolumn{5}{|c|}{ DAI } & \multirow{2}{*}{ CV (\%) } & \multirow{2}{*}{ Equação de regressão } \\
\hline & 10 & 20 & 30 & 40 & Média & & \\
\hline \multirow[t]{2}{*}{$<4 \mathrm{~mm}$} & $6,04 \mathrm{c}$ & $5,96 \mathrm{c}$ & $5,66 \mathrm{~d}$ & $5,80 \mathrm{~b}$ & 5,87 & 2,16 & $y=5,63+0,01 x-0,0005 x^{2}$ \\
\hline & & & & & & & $\mathrm{R}^{2}: 0,83^{*} *$ \\
\hline \multirow[t]{2}{*}{$4-2 \mathrm{~mm}$} & $6,18 b$ & $6,41 \mathrm{a}$ & $5,97 c$ & $5,89 b$ & 6,11 & 1,67 & $y=6,53-0,02 x^{2}$ \\
\hline & & & & & & & $\mathrm{R}^{2}: 0,98^{*} *$ \\
\hline \multirow[t]{2}{*}{$<2 \mathrm{~mm}$} & $6,38 \mathrm{a}$ & $6,20 \mathrm{~b}$ & $5,61 \mathrm{~d}$ & $5,53 \mathrm{c}$ & 5,93 & $\mathrm{I}, 6 \mathrm{I}$ & $y=7,13-0,04 x+0,0003 x^{2}$ \\
\hline & & & & & & & $\mathrm{R}^{2}: 1,00$ *** \\
\hline \multirow[t]{2}{*}{$2-1 \mathrm{~mm}$} & $6,26 \mathrm{~b}$ & $6,43 \mathrm{a}$ & $6,35 \mathrm{~b}$ & $6,16 \mathrm{a}$ & 6,3 & $\mathrm{I}, 24$ & $y=7,48-0,06+0,0008 x^{2}$ \\
\hline & & & & & & & $\mathrm{R}^{2}: 1,00$ *** \\
\hline \multirow[t]{2}{*}{$<\mathrm{Imm}$} & $6,40 \mathrm{a}$ & $6,44 \mathrm{a}$ & $6,54 \mathrm{a}$ & $6,12 \mathrm{a}$ & 6,38 & I,7I & $y=7,68-0,067 x+0,0008 x^{2}$ \\
\hline & & & & & & & $\mathrm{R}^{2}: 1,00$ *** \\
\hline
\end{tabular}

Médias seguidas da mesma letra na coluna não diferem entre si pelo teste de Tukey. NS: não significativo ao nível de $5 \%$ de probabilidade. *,** significativo a $5 \%$ e $1 \%$ de probabilidade. D: doses de $\mathrm{CaCO}_{3}$; S: substrato. I: $>4 \mathrm{~mm}, 2: 4-2 \mathrm{~mm}, 3:<2 \mathrm{~mm}, 4: 2-1$ mm, 5: <I mm. Fonte: Botucatu, SP. 2009.

Independente da dose de calcário aplicada ao substrato com menor granulometria $(<1 \mathrm{~mm})$, os valores de pH situaram-se na faixa de 5,94 a 7,2I (Tabela 2). No início da incubação, as maiores doses, de 20 e 25 $\mathrm{g} \mathrm{L}^{-1}$ apresentaram valores de $\mathrm{pH}$ entre 7,0 e 7,2I, superiores ao recomendado para a maioria das culturas, como para soja, feijão, milho e trigo está em torno de 6,0 (FAGERIA; ZIMMERMANN, 1998), para arroz de terras altas de 5,0 a 5,5 (FAGERIA, 2000), e de 5,5 a 6,5 para gérbera (ROGERS; TJIA, 1990). Inicialmente, os valores de $\mathrm{pH}$ foram mais elevados, devido a neutralização do $\mathrm{H}^{+}$presente na solução do substrato. Ao final de $40 \mathrm{DAl}$, a dose de $5 \mathrm{~g} \mathrm{~L}^{-1}$ atingiu pH inferior a 6,0. De acordo com Bailey, Fonteno e Nelson (2004), o intervalo ideal de $\mathrm{pH}$ para substratos orgânicos sem solo é 5,4 a 6,8.

De forma geral, os maiores valores de $\mathrm{pH}$ foram obtidos nos substratos com as menores granulometrias, de $<\mathrm{I} \mathrm{mm}$ e de $\mathrm{I}-2 \mathrm{~mm}$, com valores médios de 6,38 e 6,30, respectivamente (Tabelas 2 e 3). Resultado semelhante também foi encontrado por Ludwig, Guerrero e Fernandes (20I4), ao observarem que substratos com maiores proporções de casca de pinus fina, resultaram em valores de $\mathrm{pH}$ superiores, pois a menor granulometria indica um estágio de decomposição avançado, com maior estabilidade.

Visto que os valores de $\mathrm{pH}$ serão superiores e ainda não estabilizados na primeira semana após a aplicação do calcário (Tabela 3), para que possam garantir valores adequados para as culturas, recomenda-se que o plantio ocorra após 20 dias da incubação. Em aplicação de calcário com composição de $32,7 \%$ de $\mathrm{CaO}$ e 7,4\% de $\mathrm{MgO}$ em um Latossolo, se concluiu que o tempo necessário de incubação para neutralização da acidez é de 35 dias (DUDA; SALVIANO, 2007).

Os resultados obtidos permitem inferir que substratos a base de casca de pinus, com granulometrias diferenciadas devem receber quantidades de calcário distintas para que se possa obter valores ideais de $\mathrm{pH}$ para o desenvolvimento das culturas (Tabela 2). $\mathrm{O}$ pH dos substratos deve ser distinto para atender as diferentes necessidades de cada espécie (BAILEY; FONTENO; NELSON, 2004).

\section{Considerações Finais}

Substratos de casca de pinus com granulometrias diferenciadas devem receber quantidades de calcário distintas para adequar os valores de $\mathrm{pH}$ a cada cultivo. Não é recomendado utilizar o substrato na primeira semana após a aplicação do calcário devido a instabilidade do pH.

\section{Agradecimentos}

Os autores agradecem à Coordenação de Aperfeiçoamento Pessoal de Nível Superior - CAPES e ao Conselho Nacional de Desenvolvimento Científico e Tecnológico - CNPq. 


\section{Referências}

ABREU, M.F.; ABREU, C.A.; SARZI, I.; PADUA JUNIOR, A.L. Extratores aquosos para a caracterização química de substratos para plantas. Horticultura Brasileira, v. 25, n. 2, p. 184-187, 2007.

ARAÚJO, S. R.; DEMATTÊ, J. A. M.; GARBUIO, F. J. Aplicação de calcário com diferentes graus de reatividade: alterações químicas no solo cultivado com milho. Revista Brasileira de Ciência do Solo, v. 33, n. 6, p. I755-I764, 2009.

ASSIS, A. M.; FARIA, R. T.; COLOMBO, L. A.; CARVALHO, J. F. R. P. Utilização de substratos à base de coco no cultivo de Dendobrium nobile Lindl. (Orchidaceae). Acta Scientiarum Agronomy, v. 27, n. 2, p.255-260, 2005.

BAILEY, D. A.; BILDERBACK, T. Alkalinity control for irrigation water used in nurseries and greenhouses. 1997. Disponível em: https://content.ces.ncsu.edu/alkalinity-control-for-irrigation-water-used-in-nurseries-and-greenhouses. Acesso em: 30 de set. 2018.

BAILEY, D. A; FONTENO W. C; NELSON, P. V. Greenhouse substrates and fertilization. Raleigh: North Caroline State University, 2004. 15 p.

BOARO, V.; SCHWARZ, S. F.; SOUZA, P. V. D.; SOARES, W.; LOUROSA, G. V. Enxofre elementar no manejo do pH de substrato orgânico alcalino. Ciência Rural, v. 44, n. I2, p.2 I I I-2 I I7, 20 I4.

BRASIL. Instrução Normativa n. I7, de 2 I de maio de 2007. Aprova os Métodos Analíticos Oficiais para Análise de Substratos e Condicionadores de Solos. Diário Oficial da União, Brasília, 24 maio. 2007. Seção I, p.8.

CABALLERO, R.; ORDOVÁS, K.; PAJUELO, P.; CARMONA, E.; DELGADO, A. Iron chlorosis in gerbera as related to properties of various types of compost used as growing media. Communications in Soil Science and Plant Analysis, v. 38, n. 17-18, p. 2357-2369, 2007.

CAIRES, E. F; PEREIRA FILHO, P. R. S.; ZARDO FILHO, R.; FELDHAUS, I. C. Soil acidity and aluminium toxicity as affected by surface liming and cover oat residues under a no-till system. Soil Use and Management, v. 4, n. 3, p.302$309,2008$.

CAVINS, T. J.; WHIPKER, B. E.; FONTENO, W. C.; HARDEN, B.; McCALL, I.; GIBSON, J. L. Monitoring and managing pH and EC using the PourThru extraction method. Raleigh: Horticulture Information, 2000. 17 p.

CHAVES, L. H. F; FARIAS, C. H. A. Escória de siderurgia e calcário na correção da acidez do solo e na disponibilidade de cálcio, magnésio e fósforo. Revista Caatinga, v. 2 I, n. 5, p. 75-82, 2008.

DE BOODT, M.; VERDONCK, O. The physical properties of the substrates in horticulture. Acta Horticulturae, v. I, p.37-44, 1972.

DUDA, G. P.; SALVIANO, A. M. Alterações na fertilidade de um latossolo devido à prática da calagem recomendada pelo método de incubação. Revista Científica Rural, v. I2, n. I, p.28-35, 2007.

El-JAOUAL, T.; COX, D.A. Manganese toxicity in plants. Journal of Plant Nutrition, v.2I, n.2, p. 353- 386, 1998.

FAGERIA, N. K. Resposta de arroz de terras altas à correção de acidez em solo de cerrado. Pesquisa Agropecuária Brasileira, v. 35, n. II, p. 2303-2307, 2000.

FAGERIA, N. K.; ZIMMERMANN, F. J. P. Influence of pH on growth and nutrient uptake by crop species in an Oxisol. Communications in Soil Science and Plant Analysis, v. 29, n. 17, p. 2675- 2682, 1998.

FERREIRA, D. F. Sisvar: a computer statistic analysis system. Ciência e Agrotecnologia, v. 35, n. 6, p. I039- I 042 , 20 I I.

FRADE JUNIOR, E. F;; BRITO, E. S.; ORTEGA, G. P.; MATTAR, E. P. L. Neutralização química de acidez em solos sedimentares da Amazônia Ocidental, Acre. Enciclopédia Biosfera - Centro Científico Conhecer, v. 9, n. 16, p. 15661572, 2013.

HANDRECK, K. A.; BLACK, N. D. Growing Media for ornamental plants and turf. Sydney: UNSW Press, 1999.448 p. HANDRECK, K. A.; BLACK, N. D. Growing media for ornamental plants and turf. Sydney: UNSW Press, 2002.542 p. KRATZ, D.; WENDLLING, I.; NOGUEIRA, A. C.; ZOUZA, P. V. Propriedades físicas e químicas de substratos renováveis. Revista Árvore, v. 37, n. 6, p. I I03-III3, 2013.

KRATZ, D.; WENDLING, I. Produção de mudas de Eucalyptus dunniiem substratos renováveis. Floresta, v. 43, n. I, p. I25-I36, 2013. 
KÄMPF, A. N. Preparo do substrato para a produção de plantas ornamentais. In: KÄMPF, A. N. Produção comercial de plantas ornamentais. Guaíba: Agropecuária, 2005. p. 45-88.

LONE, A. B.; BARBOSA C. M.; TAKAHASHI, L. S. A.; FARIA, R. T. Aclimatização de Cattleya (Orchidaceae), em substratos alternativos ao xaxim e ao esfagno. Acta Scientiarum Agronomy, v. 30, n. 4, p. 465-469, 2008.

LUDWIG, F.; GUERRERO, A. C.; FERNANDES, D. M.; VILLAS BÔAS, R. L. Análise de crescimento de gérbera de vaso conduzida em diferentes substratos. Horticultura Brasileira, v. 28, n. I, p. 70-74, 2010.

LUDWIG, F.; FERNANDES, D. M.; GUERRERO, A. C.; VILLAS BÔAS, R. L. Características dos substratos na absorção de nutrientes e na produção de gérbera de vaso. Horticultura Brasileira, v. 32, n. 2, p. I84-I89, 2014.

LUDWIG, F; GUERRERO, A. C.; FERNANDES, D. M. Caracterização física e química de substratos formulados com casca de pinus e terra de subsolo. Cultivando o Saber, v. 7, n. 2, p. I52-162, 2014.

MERCURIO, G. Gerbera cultivation in greenhouse. The Netherlands: Schreurs, 2002. 206p.

NASCENTE, A. S.; COBUCCI, T. Calcário na forma de micropartículas aplicado no sulco de semeadura aumenta produtividade do feijoeiro. Revista Ceres, v. 62, n. 6, p. 597-606, 2015.

POLETTO, I.; MUNIZ, M. F. B.; CECONI, D. E.; BLUME, E. Influência da aplicação de NPK e calcário sobre o crescimento da erva mate, severidade da podridão-de-raízes e população fúngica do solo. Ciência Florestal, v. 2I, n. 3, p. 429-444, 2011.

ROGERS, M. N.; TJIA, B. O. Gerbera production for cut flowers and pot plants. Portland: Timber Press, 1990. II6p. SAMPAIO, R. A.; RAMOS, S. J.; GUILHERME, D. O.; COSTA, C. A.; FERNANDES, L. A. Produção de mudas de tomateiro em substratos contendo fibra de coco e pó de rocha. Horticultura Brasileira, v. 26, n. 4, p. 499-503, 2008.

SOUSA, L. F. R. A.; MARINHO, R. W. D.; NUNES, F. M.; SILVA, R. V.; NASCIMENTO, I. O.; SILVA, W. A. Determinação do $\mathrm{pH}$ de um argissolo vermelho amarelo distrófico incubado com aplicação de doses crescentes de $\mathrm{CaCO} 3$ por diferentes métodos. Revista Agroecossistemas, v. 5, n. 2, p. 58-63, 2013.

SONNEVELD, C.; VOOGT, W. Effects of $\mathrm{pH}$ and $\mathrm{Mn}$ application on yield and nutrient absorption with rockwool grown gerbera (refereed). Acta Horticulturae, v. 450, p. 139-147, 1997.

VElOSO, C. A. C.; BORGES, A. L.; MUNIZ, A. S.; VEIGAS, I. A. J. M. Efeito de diferentes materiais no pH do solo. Scientia Agricola, v. 49, n. I, p. I23-128, 1992. 